\title{
Intramolecular Hydrogen Bonding in Isotactic Poly(methacrylamide)s and Its Implications for Control of Side-Chain Orientation
}

\author{
Takayuki Nakahira, ${ }^{\dagger}$ Fan Lin, ${ }^{*}$ Cham Tau Boon, Takeshi Karato, Masahiko AnnakA, \\ Masako YoshIKuni, and Susumu IWABUCHI
}

Department of Applied Chemistry, Faculty of Engineering, Chiba University,

Yayoi-cho 1-33, Inage-ku, Chiba 263, Japan

(Received February 20, 1997)

KEY WORDS Poly(methacrylamide) / Intramolecular Hydrogen Bonding / Secondary Structure /
Circular Dichroism /

The photoprocesses of polymers differ from those of isolated low-molecular-weight analogues ${ }^{1}$ : excitation energy can be transported in the polymer through the pendant chromophores as in crystals or concentrated solutions of low-molecular-weight analogues ${ }^{2}$. Excitation energy, however, can be trapped as excimers (excited dimers) which are formed with pairs of chromophores suitably situated for their formation along the polymer chain. The chromophore interactions in the excited state, i.e., excitation energy transport and trapping, are affected by the stereoregularity and conformation of the main chain $^{3}$ as well as by the steric hindrance introduced in the side chain. ${ }^{4}$ While it is not fully understood how these structural differences actually affect energy migration and excimer formation, one may be able to enhance the former and suppress the latter if one properly controls the orientation of the chromophores along the polymer chain. If this is possible, "a molecular wire" will be obtained which allows efficient one-dimensional transport of excitation energy along the polymer chain. Studies along this line have been carried out using polypeptides where side-chain chromophores are arranged at regular intervals along the helical main chain. ${ }^{5}$

Previously, we have prepared poly(L-glutamines) where naphthalene chromophores are introduced in the side chain via amide linkage and examined how the sidechain structure affects the hydrogen bonding interactions among the side-chain amide groups and subsequent ordering of the side-chain chromophores. ${ }^{6}$ In the present study we prepared isotactic and atactic polymethacrylamides, i.e., poly(1-naphthylmethyl methacrylamide) (1) and poly[(S)-1-(1-naphthyl)ethyl methacrylamide] (2), where naphthalene chromophores are likewise introduced in the side chains via amide linkage and examined how the stereoregularity and the side-chain structure affect the hydrogen bonding interactions among the side-chain amide groups and the subsequent orientation of the side-chain chromophores.<smiles>[2H]NC(=O)C(C)(CC(C)(C)C)C(F)(F)F</smiles>

\section{EXPERIMENTAL}

\section{Monomeric Model Compound}

(S)-1-Naphthylethylacetamide, was prepared from the corresponding amine and acetyl chloride, purified by recrystallization from a mixed solvent of petroleum ether and benzene, its structure being confirmed by ${ }^{1} \mathrm{H}$ NMR.

\section{Polymers}

Isotactic poly(methyl methacrylate) (PMMA) having an isotactic triad content of larger than $99 \%$ as determined by ${ }^{1} \mathrm{H}$ NMR was prepared from the monomer at room temperature in toluene using phenyl magnesium bromide as an anionic initiator. ${ }^{7}$ Atactic PMMA was synthesized in tetrahydrofuran (THF) at $60^{\circ} \mathrm{C}$ using azobisisobutyronitrile (AIBN) as a radical initiator, its isotactic, heterotactic, and syndiotactic triad contents being 2,32 , and $64 \%$, respectively. The polymers precipitated in methanol were subsequently purified by repeated precipitation from chloroform to methanol. The degrees of polymerization of isotactic and atactic PMMA, determined on the basis of standard PMMA samples by gel permeation chromatography using THF as a solvent, were 440 and 700 , respectively.

The PMMA samples, freeze-dried from benzene, were dissolved in concentrated sulfuric acid. The solution was stirred at $45^{\circ} \mathrm{C}$ for 2 hours in the case of isotactic PMMA and at room temperature for 6 days in the case of atactic PMMA. After precipitated in water, the polymer was treated with boiling water for 24 hours to hydrolyze any acid anhydride present. ${ }^{8}$ The polymers were shown to be completely hydrolyzed by IR and ${ }^{1} \mathrm{H}$ NMR.

Poly(methacrylamide)s were prepared by condensation

\footnotetext{
† To whom correspondence should be addressed.

* Graduate School of Science and Technology, Chiba University, Yagyoi-cho 1-33, Inage-ku, Chiba 263, Japan.
} 
of the polymethacrylic acid with amines in DMAc using dicyclohexylcarbodiimide/1-hydroxybenzotriazole as a condensing agent. ${ }^{9}$ The amidation was confirmed to be close to $100 \%$ by elemental analysis and ${ }^{1} \mathrm{H}$ NMR.

\section{Measurements}

The IR and ${ }^{1} \mathrm{H}$ NMR spectra were recorded on a JASCO JIR-7000 FT-IR spectrophotometer and on a JEOL GSX-500 ${ }^{1} \mathrm{H}$ NMR spectrometer, respectively. CD measurements were carried out using a JASCO J-500 circular dichrograph.

\section{RESULTS AND DISCUSSION}

The IR spectra of isotactic and atactic $\mathbf{1}$ and $\mathbf{2}$ as well as that of the monomeric model compound in chloroform are shown in Figures 1 and 2. It is well established that the absorption at $c a .3450 \mathrm{~cm}^{-1}$ is due to the stretching vibration of amide $\mathrm{N}-\mathrm{H}$ (amide $\mathrm{A}$ band) free from hydrogen bonding and that at $c a .3320 \mathrm{~cm}^{-1}$ is due to that of hydrogen-bonded amide $\mathrm{N}-\mathrm{H}$. The broadness of the hydrogen-bonded amide $\mathrm{N}-\mathrm{H}$ band reflects, in large part, a distribution of hydrogen-bonded $\mathrm{N}-\mathrm{H}$ groups of varying strength dictated by distance and geometry. ${ }^{10}$ As is seen with atactic $\mathbf{1}$ and $\mathbf{2}$, there appears to be an absorption around $3380 \mathrm{~cm}^{-1}$, due to partially hydrogenbonded $\mathrm{N}-\mathrm{H}$ group. ${ }^{11}$ Similarly, the $\mathrm{C}=\mathrm{O}$ stretching band (amide I band), which appears in the 1600-1700 $\mathrm{cm}^{-1}$ region, suggests the presence of fully hydrogenbonded $\left(\mathrm{ca} .1620 \mathrm{~cm}^{-1}\right)$, partially hydrogen-bonded ( $c a$. $\left.1640 \mathrm{~cm}^{-1}\right)$, and free $\left(\mathrm{ca} .1660 \mathrm{~cm}^{-1}\right)$ amide carbonyl

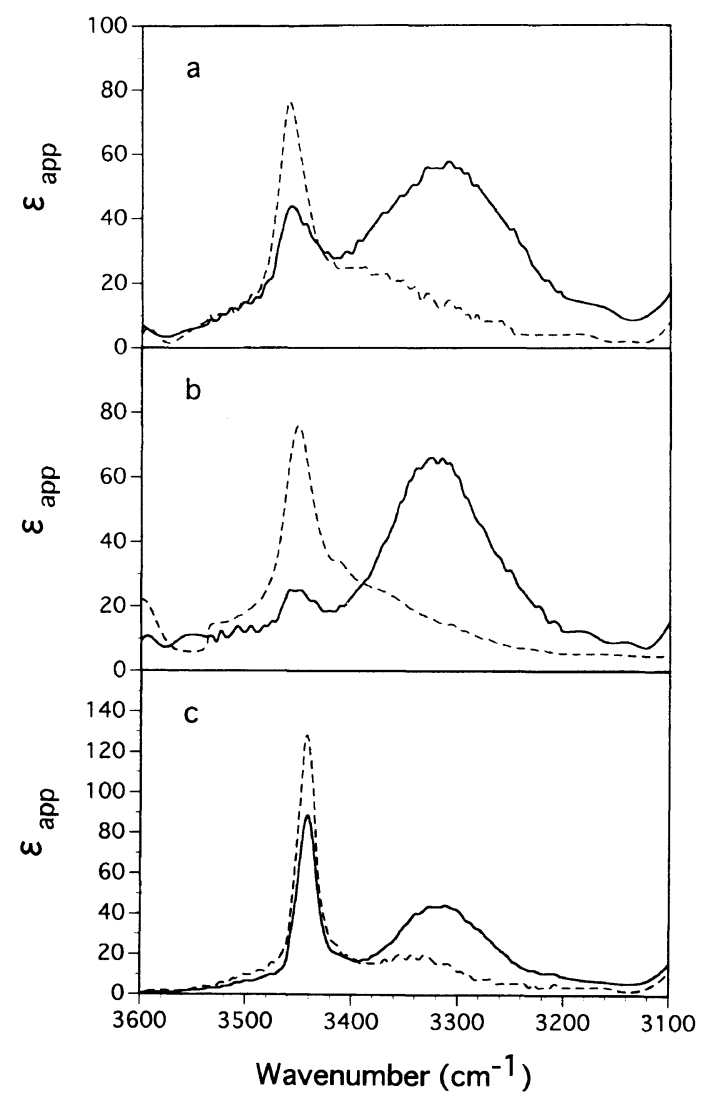

Figure 1. Amide A bands of (a) $0.05 \mathrm{M}$ isotactic (- - and atactic (--) 1, (b) $0.05 \mathrm{M}$ isotactic (-) and atactic (--) 2, and (c) $0.5 \mathrm{M}$ $(-)$ and $0.01 \mathrm{M}(---)(S)$-1-naphthylethylacetamide in chloroform. groups. Unlike that of the monomeric model compound, the amide $\mathrm{A}$ bands of the poly(methacrylamide)s do not change in the concentration range of $0.01-0.5 \mathrm{M}$ (in terms of repeating unit); indicating that the sidechain amide groups of the polymers are intramolecularly hydrogen-bonded. Some intermolecular association should occur in concentrated solutions such as those used for IR measurements. Intermolecular hydrogen bonding, however, does not appear to compete with intramolecular hydrogen bonding as is also the case with polyacrylamide in water. ${ }^{12}$ The side-chain amide groups of the isotactic polymers are thus shown to form intramolecular hydrogen bonds rather extensively while those of the atactic polymers form such bonds only partially. Furthermore, it can be seen that the extent of intramolecular hydrogen bonding is greater, with a narrower distribution, in isotactic $\mathbf{2}$ as compared with that in isotactic 1, suggesting a favorable steric effect of methyl substituents in the side chains in intramolecular hydrogen bonding. This favorable steric effect is also seen in the IR spectra taken in a moderately hydrogenbonding solvent such as dioxane: most of the intramolecular hydrogen bonding of isotactic $\mathbf{2}$ is maintained in dioxane as in chloroform while that of isotactic $\mathbf{1}$ is lost significantly due to the hydrogen-bonding solvation of the amide $\mathrm{N}-\mathrm{H} .{ }^{13}$

The ${ }^{1} \mathrm{H}$ NMR spectra of the polymers are shown in Figure 3. Amide protons free from hydrogen bonding and those hydrogen-bonded are known to give signals at $c a .6$ and $8 \mathrm{ppm}$, respectively, in non-hydrogen-bonding solvents such as $\mathrm{CDCl}_{3}$. Atactic 1 gives a signal

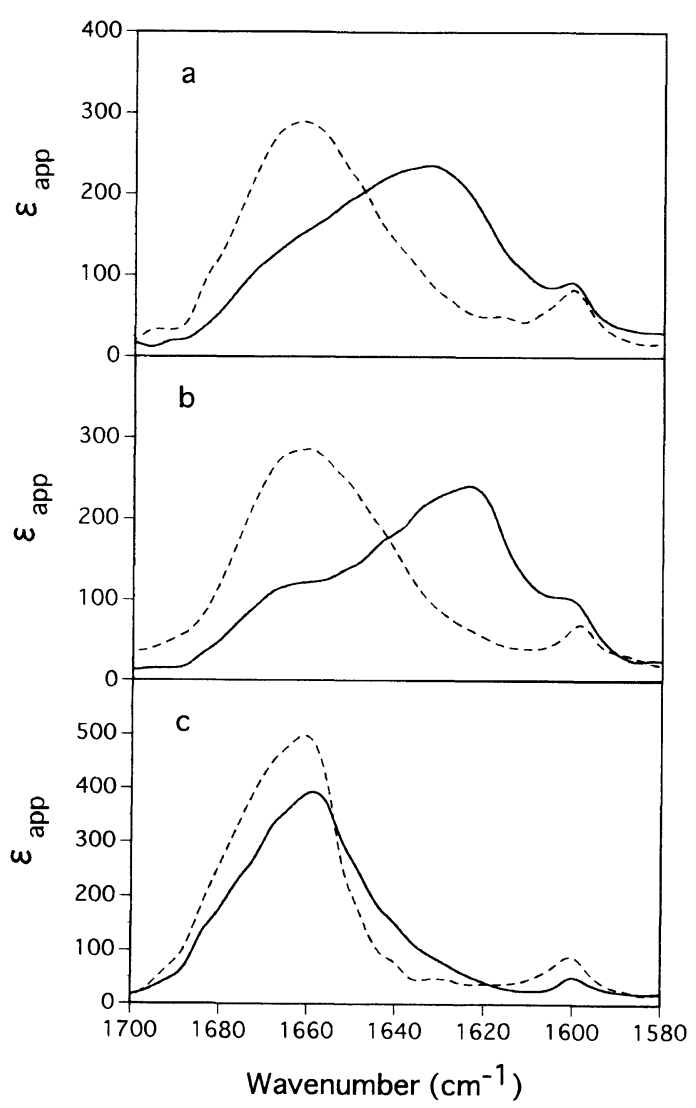

Figure 2. Amide I bands of (a) $0.05 \mathrm{M}$ isotactic ( - ) and atactic (--) 1, (b) $0.05 \mathrm{M}$ isotactic $(-)$ and atactic (--) 2, and (c) $0.5 \mathrm{M}$ $(-)$ and $0.01 \mathrm{M} \mathrm{(---)}(S)$-1-naphthylethylacetamide in chloroform. 


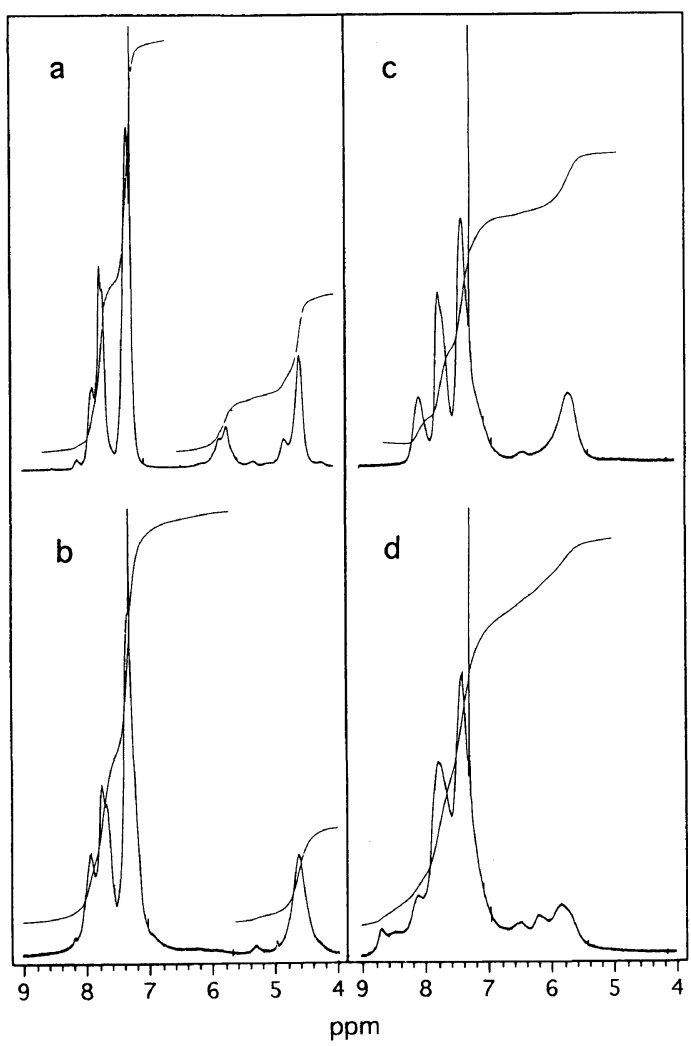

Figure 3. $500 \mathrm{MHz}{ }^{1} \mathrm{H}$ NMR spectra of atactic (a) and isotactic (b) 1 and atactic (c) and isotactic (d) 2 in chloroform- $d_{1}$. Sharp signals at $7.2 \mathrm{ppm}$ are due to $\mathrm{CHCl}_{3}$ impurity in the solvent.

at $c a .5 .8 \mathrm{ppm}$, which accounts for about $90 \%$ of the total amide protons. In the case of isotactic 1 , the corresponding signal is much less intense and thus the hydrogen-bonded amide protons must reside in the 7$8 \mathrm{ppm}$ region where intensive aromatic proton signals appear. (Isotactic poly( $n$-propyl methacrylamide), for example, gives signals at 8.2 and $7.1 \mathrm{ppm}$ in addition to a signal at $6.0 \mathrm{ppm}$, suggesting the presence of fully hydrogen-bonded, partially hydrogen-bonded, and free amide protons. ${ }^{13}$ )

In the case of atactic 2, most of the amide protons are free from hydrogen bonding and appear at $c a .5 .8 \mathrm{ppm}$, overlapping with the methine protons in the side chains. Isotactic $\mathbf{2}$, on the other hand, gives a rather complex spectrum, seemingly reflecting, at least in part, a rigid orientation of the side chains with respect to each other and/or with respect to the main chain. The signals at $8.2-8.8 \mathrm{ppm}$, nevertheless, suggest the presence of strong hydrogen bonds.

Intramolecular hydrogen bonding may lead to a controlled secondary structure and thus to a controlled orientation of side-chain chromophores. The preliminary results of molecular mechanics calculation suggest that isotactic poly(methacrylamide)s form $\mathrm{ca} .5_{3}$ helical structures with hydrogen bonding occurring between the amide groups of every other monomer units along the polymer chain. Possible control of the secondary structure and side-chain orientation is thus examined by measuring the circular dichroic (CD) spectrum of isotactic 2 in 1,2-dichloroethane (EDC), EDC being used as it allows measurements down to $220 \mathrm{~nm}$. Rather strong $\mathrm{CD}$ signals with exciton splitting, observed in the ${ }^{1} \mathrm{~B}_{\mathrm{b}}$

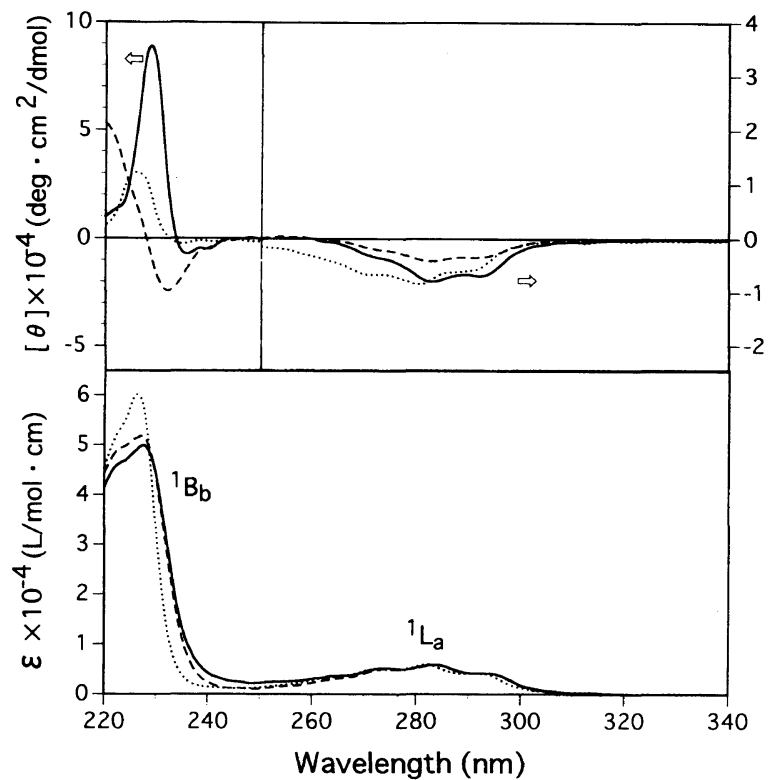

Figure 4. UV (lower curves) and CD (upper curves) spectra of isotactic (-) and atactic (--) 2 and those of $(S)$-1-naphthylethylacetamide (-----) in 1,2-dichloroethane.

band of the naphthalene chromophore, suggest formation of a regular secondary structure, e.g., one-handed helix, with rather rigidly oriented naphthalene chromophores. Additionally, the CD signal in the ${ }^{1} \mathrm{~L}_{\mathrm{a}}$ band, when compared with that of the monomeric compound, suggests partial exciton coupling interaction.

Atactic 2 also gives a CD spectrum very much different from that of the monomeric model compound; besides the obvious differences in the ${ }^{1} B_{b}$ band, the signals in the ${ }^{1} \mathrm{~L}_{\mathrm{a}}$ band are significantly diminished. This may reflect some local ordering of naphthalene chromophores, which differs from that of isotactic 2 , possibly due to the presence of partially hydrogen-bonded amide groups.

In dioxane, both isotactic and atactic 2 give $C D$ spectra similar to that of atactic 2 in EDC, the negative signal of isotactic 2 at $c a$. $230 \mathrm{~nm}$ being somewhat stronger than that of atactic 2. (Addition of trifluoroacetic acid to EDC up to $5 \mathrm{vol} \%$ brings about a similar change in the CD spectrum of isotactic 2. ${ }^{13)}$ ) It is thus suggested that hydrogen bonding solvation breaks down some of fully hydrogen-bonded amide groups and thereby disrupts the regular secondary structure.

\section{CONCLUSION}

The intramolecular hydrogen bonding interactions of the side-chain amide groups in isotactic and atactic $\mathbf{1}$ and $\mathbf{2}$ were studied by IR and ${ }^{1} \mathrm{H}$ NMR. The IR spectra recorded in chloroform showed that isotactic $\mathbf{1}$ and $\mathbf{2}$ form extensive intramolecular hydrogen bonding among the side-chain amide groups while corresponding atactic ones do so only partially. The results of ${ }^{1} \mathrm{H}$ NMR are consistent with the IR results. Isotactic and atactic $\mathbf{2}$ gave markedly different CD spectra in EDC. Rather strong CD signals observed with isotactic 2 suggest the formation of a regular secondary structure, e.g., a one-handed helix, which leads to a specific side-chain orientation.

Detailed discussion of CD spectra as well as implications of the chromophore orientation as to excitation 
energy transport along the polymer chain will be reported in a future publication.

\section{REFERENCES}

1. I. Soutar and D. Phillips, in "Photophysical and Photochemical Tools in Polymer Science," by M. A. Winnik, Ed., D. Reidel Publishing Co., Holland, 1985, p 85.

2. J. B. Birks, "Photophysics of Aromatic Molecules," Wiley Interscience, New York, N.Y., 1970.

3. T. Ishii, T. Handa, and S. Matsunaga, J. Polym. Sci., Polym. Chem. Ed., 17, 811 (1979).

4. T. Nakahira, S. Ishizuka, S. Iwabuchi, and K. Kojima, Macromolecules, 16, 297 (1983).

5. M. Sisido, S. Egusa, and Y. Imanishi, J. Am. Chem. Soc., 105,
4077 (1983).

6. M. Sato, M. Yoshimoto, T. Nakahira, and S. Iwabuchi, Makromol. Chem., Rapid Commun., 14, 179 (1993).

7. K. Hatada, K. Ute, K. Tanaka, T. Kitayama, and Y. Okamoto, Polym. J., 17, 977 (1985).

8. J. Semen and J. B. Lando, Macromolecules, 2, 570 (1969).

9. M. Sato, H. Morikawa, M. Yoshimoto, T. Nakahira, and S. Iwabuchi, Nihon Kagaku Kaishi, 1363 (1992).

10. D. J. Skrovanek, S. E. Howe, P. C. Painter, and M. M. Coleman, Macromolecules, 18, 1676 (1985).

11. M. Tsuboi, Nihon Kagaku Zasshi, 76, 376 (1955).

12. N. Tanaka, K. Ito, and H. Kitano, Macromolecules, 27, 540 (1994).

13. T. Nakahira, F. Lin, C. T. Boon, T. Karato, M. Annaka, and M. Yoshikuni, to be submitted. 